\title{
Clinical study on local application of low-dose insulin for promoting wound healing after operation for deep burns
}

\author{
MING ZENG, YAN ZHI, WENJUN LIU, WEI ZHANG and JINXIONG XU \\ Department of Burns, The Second Affiliated Hospital of Kunming \\ Medical University, Kunming, Yunnan 650101, P.R. China
}

Received April 1, 2016; Accepted September 22, 2016

DOI: $10.3892 /$ etm.2016.3749

\begin{abstract}
Transplanted free skin flaps are often needed to treat deep burns; their survival, however, is less than optimal. This study examined whether local low-dose insulin injections can promote flap survival and wound healing after surgery. A total of 165 patients who underwent free skin flap transplantation for simple deep burns were enrolled in the study and divided into 5 groups of 33 patients each: Blank control group (no local subcutaneous drug injections), saline control group (saline injections), low-dose insulin group (0.5 units regular insulin injections), medium-dose group (1.0 units regular insulin injections) and high-dose group (2.0 units regular insulin injections). Wound healing and flap survival conditions were assessed and compared among groups. The best wound healing rate found was that of the low-dose insulin injection group where all the parameters measured improved significantly: The healing time was shorter; the blood flow volume, the flap survival, the number of fibroblasts and new vessels increased; the re-epithelialization occurred faster; the infiltration of inflammatory cells was reduced; the expression levels of heat shock protein-90, vascular endothelial growth factor, transforming growth factor- $\beta$ and interleukin-1 were higher; and the plasma glucose levels only fluctuated slightly. The results clearly demonstrate that a local low-dose insulin regime after flap transplantation can accelerate the healing time and improve the surgical outcome without exerting detrimental secondary effects on the glucose plasma level of deep burn patients.
\end{abstract}

\section{Introduction}

Deep burns cause severe damage and tissue regeneration and wound healing is difficult (1). The survival of a transplanted

Correspondence to: Dr Yan Zhi, Department of Burns, The Second Affiliated Hospital of Kunming Medical University, 374 Dianmian Road, Kunming, Yunnan 650101, P.R. China

E-mail: zhi_yan1212@163.com

Key words: low-dose insulin, deep burn, wound healing, transplanted flap, heat shock protein-90, vascular endothelial growth factor, transforming growth factor- $\beta$, interleukin-1 CD31 immunohistochemistry free skin flap is closely related to local micro-vessel density, hemoperfusion and nearby cell migration (2). The stress reaction after a deep burn causes generalized resistance to insulin and a negative nitrogen balance (3). A local injection of insulin has been shown to be safe and effective in promoting wound healing while exerting little influence on the plasma glucose levels in a diabetic rat model (4). However, as studies in humans have not been conducted, there are many questions that remain unanswered and it is not even clear that a local application of low-dose insulin is beneficial for wound healing.

The aim of the present study was to examine the effects of local low-dose insulin treatment after operation for deep burns, providing valuable information and a reference for clinical use.

\section{Subjects and methods}

Subjects information. A total of 165 patients with deep burns were enrolled in the study in The Second Affiliated Hospital of Kunming Medical University (Yunnan, China) from January, 2013 to January, 2016. Exclusion criteria for the study were the presence of co-existing diseases including acute lung injury, severe infections, malnutrition, diabetes, scarring tissues, other severe underlying diseases and failed free flap transplantation. After obtaining the approval of the ethics committee of The Second Affiliated Hospital of Kunming Medical University and informed consent of patients or their relatives, the cases were divided into 5 equal size groups, by random assignment: A blank control group, a saline control group, a low-dose insulin group, median dose insulin group and a high dose insulin group. The baseline data in each group were compared to the data to the other groups and differences were considered statistically significant $(\mathrm{P}<0.05$; Table I).

Treatment method. All the patients had similar free skin flap transplantation operations performed by one nursing team, and prophylaxis for infections and strengthening of nourishments according to the standard medical procedures for burns were followed. The individuals in the blank control group had no local subcutaneous drug injections. The saline control group patients were subcutaneously injected with $2 \mathrm{ml}$ of saline once daily for 14 days. The individuals in the insulin groups received local insulin injections at a total volume of $2 \mathrm{ml}$ once a day for 14 days: The low insulin group received 
Table I. Comparison of inter-group baseline data.

\begin{tabular}{|c|c|c|c|c|c|c|c|c|c|c|c|}
\hline Group & Case & $\begin{array}{l}\text { Male/ } \\
\text { female }\end{array}$ & Age & $\begin{array}{c}\text { Course } \\
\text { of disease, } h\end{array}$ & Deep II $^{\circ}$ & $\mathrm{III}^{\circ}$ & $\begin{array}{c}\text { Extent of } \\
\text { burn (\% TBSA) }\end{array}$ & Limbs & Chest & Abdomen & Back \\
\hline Blank control & 33 & $19 / 14$ & $52.4 \pm 10.3$ & $1.2 \pm 0.4$ & 17 & 16 & $35.6 \pm 7.8$ & 16 & 3 & 4 & 10 \\
\hline Saline control & 33 & $20 / 13$ & $51.3 \pm 11.4$ & $1.3 \pm 0.5$ & 18 & 15 & $36.4 \pm 7.2$ & 18 & 2 & 2 & 11 \\
\hline Low dose & 33 & $21 / 12$ & $53.2 \pm 12.2$ & $1.4 \pm 0.7$ & 19 & 14 & $35.8 \pm 7.5$ & 17 & 3 & 3 & 10 \\
\hline Median dose & 33 & $20 / 13$ & $54.5 \pm 13.5$ & $1.1 \pm 0.6$ & 16 & 17 & $36.3 \pm 7.6$ & 15 & 4 & 2 & 12 \\
\hline High dose & 33 & 21/12 & $52.7 \pm 12.6$ & $1.3 \pm 0.5$ & 18 & 15 & $35.9 \pm 7.7$ & 16 & 5 & 3 & 9 \\
\hline $\mathrm{F}\left(\chi^{2}\right)$ & & 0.357 & 0.625 & 0.514 & 0.633 & & 0.249 & & & 3.335 & \\
\hline P-value & & 0.986 & 0.349 & 0.636 & 0.959 & & 0.302 & & & 0.993 & \\
\hline
\end{tabular}

0.5 units regular insulin injections, the median dose group had 1.0 units regular insulin injections and the high dose group had 2.0 units regular insulin injections.

Observation indexes. Wound healing and flap survival conditions were assessed with a variety of methods and compared amongst groups.

The healing rate of wounds was determined by using a transparent film to manually trace the wound outline and then its area calculated with the imageJ software using the formula: Healing rate $=$ area of wound healed/total area $\times 100 \%$.

The flap survival in each case was studied taking digital images of the flap with a camera (Canon, Inc., Tokyo, Japan), and then calculating the survival rate using the image analysis software image-Pro Plus.v 6.0 (Microsoft Corporation, Redmond, WA, USA). The survival rate was calculated as: survival areas/design area $\mathrm{x} 100 \%$.

The wound blood flow volumes were measured by laser doppler flowmetry according to a previous study (5).

Skin flap biopsies of each healed-unhealed interface tissue $(0.5 \times 0.5 \times 0.5 \mathrm{~mm})$ were processed for hematoxylin and eosin staining and histological observation by conventional fixation, embedding and sectioning.

CD31 immunohistochemical detection in biopsies was used to measure microvessel quantity. Briefly, sample tissues were incubated overnight at $4^{\circ} \mathrm{C}$, with rabbit polyclonal anti-CD31 antibody (Abcam, Cambridge, MA, USA; catalog no.: ab28364; dilution: 1:50). After washing 3 times with phosphate-buffered saline, the samples were incubated for $1 \mathrm{~h}$ goat anti-rabbit monoclonal tetramethylrhodamine isothiocyanate-labelled secondary antibody (Abcam; catalog no.: ab6718; dilution: 1:2000). Cell nuclei were dyed using DAPI. A laser scanning confocal microscope (Lucid Inc., Rochester, NY, USA) was used to observe the samples and estimate the blood vessel density by counting the number of vessels in five sections and working out an average number.

The expression levels of heat shock protein (HSP)-90, vascular endothelial growth factor (VEGF), transforming growth factor (TGF) $-\beta$ and interleukin (IL)- 1 were evaluated using an ELISA kit strictly adhering to the manufacturer's instructions (R\&D Systems, Inc., Minneapolis, MN, USA).

Conventional biochemical methods were used to detect the levels of glucose in plasma.
Statistical analysis. SPSS 19.0 (SPSS, Inc., Chicago, IL, USA) was used for statistical analysis. Numerical data were expressed as mean \pm standard deviation. For comparison between groups, single-factor ANOVA analysis was selected, while pairwise comparisons were made by the least significant difference test. Categorical data were analyzed with the Chi-square test and expressed as number of cases and percentage. $\mathrm{P}<0.05$ was considered to indicate a statistically significant difference.

\section{Results}

Comparisons of wound healing and flap survival conditions. The wound healing time of the low-dose insulin group was significantly shorter while the high-dose insulin group took the longest time to heal, and differences were statistically significant $(\mathrm{P}<0.05)$. The healing rate at 14 days post-intervention was higher than that after 7 days for all groups, however, the healing rate for the high dose insulin group was the lowest of them all, indicating that a high dose of insulin actually impaired normal healing (Table II).

The total flap survival rate in the low-dose insulin group was the highest while the high-dose insulin group had the lowest survival rate of all the groups, and differences were statistically significant $(\mathrm{P}<0.05)$. There were no significant differences between the survival rates for the blank control, saline control, and high-dose insulin groups. The survival rate for the low-dose and medium-dose insulin groups was higher at 14 days than at 7 days.

Comparison of wound blood flow volume. The blood flow volume in the low-dose insulin group was the best of them all after 14 days, while that of the high-dose insulin level was the worst. The flows were superior after 14 days than after 7 days for all the groups, and the differences were statistically significant (Fig. 1).

Histological observation of wound healing. After 7 days of treatment the low-dose insulin group cases had a reduced number of inflammatory cells in the wound, the fibroblast counts had increased, the capillary components had increased, and partial regenerated epithelial tissue was observed. By contrast, the high-dose insulin group had the highest number of inflammatory cells present in the wound 
Table II. Comparison between wound healing and flap survival conditions.

\begin{tabular}{|c|c|c|c|c|c|c|}
\hline Group & $\begin{array}{l}\text { Wound healing } \\
\text { time, days }\end{array}$ & $\begin{array}{c}7 \text { days healing } \\
\text { rate, } \%\end{array}$ & $\begin{array}{c}14 \text { days healing } \\
\text { rate, } \%\end{array}$ & $\begin{array}{c}\text { Total flap } \\
\text { survival rate, } \%\end{array}$ & $\begin{array}{c}7 \text { days } \\
\text { survival rate, } \%\end{array}$ & $\begin{array}{c}14 \text { days } \\
\text { survival rate, } \%\end{array}$ \\
\hline Blank control & $27.5 \pm 4.2$ & $47.2 \pm 10.3$ & $53.9 \pm 11.2$ & $70.5 \pm 6.9$ & $61.5 \pm 7.2$ & $68.5 \pm 7.4$ \\
\hline Saline control & $26.6 \pm 4.5$ & $48.8 \pm 11.2$ & $55.7 \pm 11.6$ & $72.3 \pm 6.8$ & $63.2 \pm 7.3$ & $69.7 \pm 7.5$ \\
\hline Low dose & $18.2 \pm 3.3$ & $72.8 \pm 8.6$ & $86.5 \pm 10.4$ & $92.5 \pm 5.4$ & $83.4 \pm 6.3$ & $90.6 \pm 6.6$ \\
\hline Medium dose & $22.4 \pm 5.2$ & $56.9 \pm 12.3$ & $70.3 \pm 12.6$ & $78.8 \pm 6.6$ & $72.1 \pm 7.8$ & $76.5 \pm 7.3$ \\
\hline High dose & $31.3 \pm 5.6$ & $33.4 \pm 14.2$ & $48.4 \pm 15.5$ & $67.9 \pm 6.3$ & $64.4 \pm 7.1$ & $65.3 \pm 7.2$ \\
\hline $\mathrm{F}$ & 8.245 & 9.654 & 10.772 & 7.648 & 8.524 & 8.639 \\
\hline P-value & $<0.001$ & $<0.001$ & $<0.001$ & $<0.001$ & $<0.001$ & $<0.001$ \\
\hline
\end{tabular}

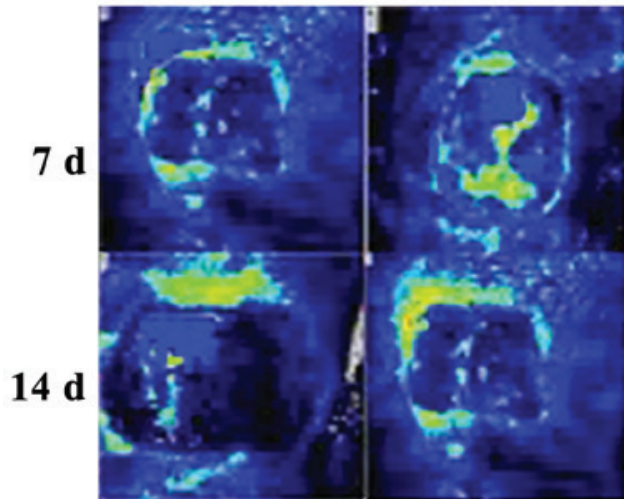
$\underset{\text { group }}{\operatorname{Brank} \text { control }} \underset{\text { group }}{\text { Saline control }}$
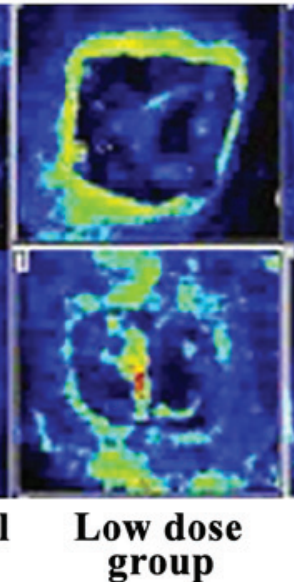

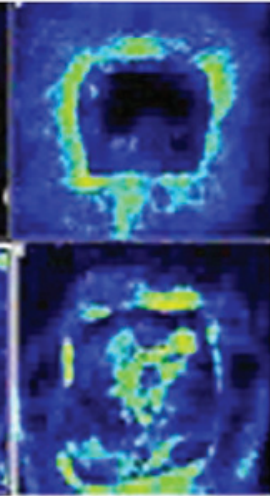

Median dose group

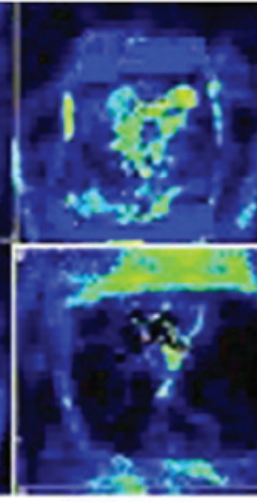

High dose group

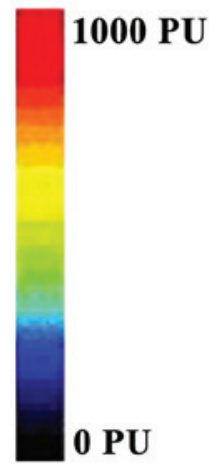

PU

Figure 1. Flowmetry of representative biopsy tissues from different groups at 7 and 14 days.

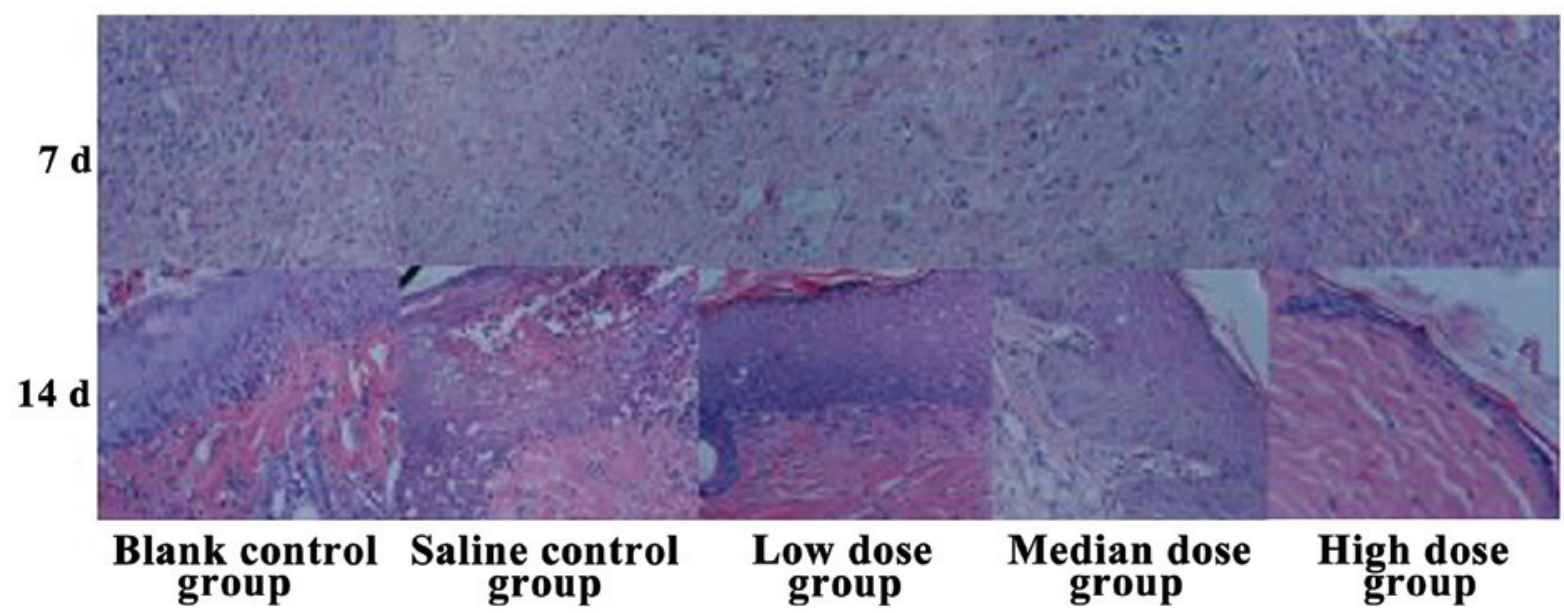

Figure 2. Microscopic (magnification, x100) images of representative biopsy tissues from each group at 7 and 14 days.

and the least counts of fibroblasts. Furthermore, after 14 days, the low-dose insulin group showed a regenerated epidermal structure, rete pegs were clearly visible at the epidermal and dermal attachment sites, new vessels increased, and fibroblast cells were arranged in neat rows. By contrast, the high-dose insulin group had fewer regenerated epidermal structures that were too fragile to cover all the epidermal layers, albeit with abundant inflammatory cell infiltration (Fig. 2).

Comparison of expression levels of HSP-90, VEGF, TGF- $\beta$ and $I L-1$. Expression levels of HSP-90, VEGF, TGF- $\beta$ and IL-1 at 7 days were higher than those at 14 days for all groups, however, they were highest in the low-dose insulin group. The 
Table III. Expression levels of HSP-90, VEGF, TGF- $\beta$ and IL-1.

\begin{tabular}{|c|c|c|c|c|c|c|c|c|}
\hline \multirow[b]{2}{*}{ Group } & \multicolumn{2}{|c|}{ HSP-90, ng/ml } & \multicolumn{2}{|c|}{ VEGF, $\mu \mathrm{g} / \mathrm{ml}$} & \multicolumn{2}{|c|}{ TGF- $\beta, p g / m l$} & \multicolumn{2}{|c|}{ IL-1, pg/ml } \\
\hline & 7 days & 14 days & 7 days & 14 days & 7 days & 14 days & 7 days & 14 days \\
\hline Blank control & $25.2 \pm 6.4$ & $16.8 \pm 6.5$ & $14.3 \pm 8.2$ & $12.9 \pm 8.4$ & $45.9 \pm 15.2$ & $24.8 \pm 11.2$ & $73.2 \pm 32.6$ & $39.7 \pm 14.2$ \\
\hline Saline control & $26.3 \pm 6.9$ & $17.2 \pm 6.8$ & $15.2 \pm 7.6$ & $13.5 \pm 7.8$ & $44.8 \pm 14.7$ & $26.5 \pm 11.4$ & $75.7 \pm 36.7$ & $36.5 \pm 13.9$ \\
\hline Low dose & $63.4 \pm 12.3$ & $42.5 \pm 12.6$ & $44.8 \pm 10.3$ & $36.5 \pm 10.5$ & $156.3 \pm 43.2$ & $85.7 \pm 36.5$ & $247.3 \pm 62.4$ & $112.3 \pm 48.5$ \\
\hline Medium dose & $52.6 \pm 13.7$ & $33.7 \pm 13.9$ & $32.8 \pm 11.2$ & $27.6 \pm 11.3$ & $120.5 \pm 45.7$ & $64.5 \pm 32.4$ & $215.8 \pm 70.2$ & $86.4 \pm 36.5$ \\
\hline High dose & $24.8 \pm 6.6$ & $15.5 \pm 6.3$ & $14.4 \pm 7.2$ & $12.7 \pm 7.3$ & $43.2 \pm 15.8$ & $23.9 \pm 10.3$ & $69.8 \pm 35.5$ & $37.2 \pm 12.5$ \\
\hline $\mathrm{F}$ & 10.523 & 8.659 & 8.645 & 9.203 & 15.426 & 13.258 & 14.527 & 13.625 \\
\hline P-value & $<0.001$ & $<0.001$ & $<0.001$ & $<0.001$ & $<0.001$ & $<0.001$ & $<0.001$ & $<0.001$ \\
\hline
\end{tabular}

HSP-90, heat shock protein-90; VEGF, vascular endothelial growth factor; TGF- $\beta$, transforming growth factor- $\beta$; IL-1, interleukin-1.

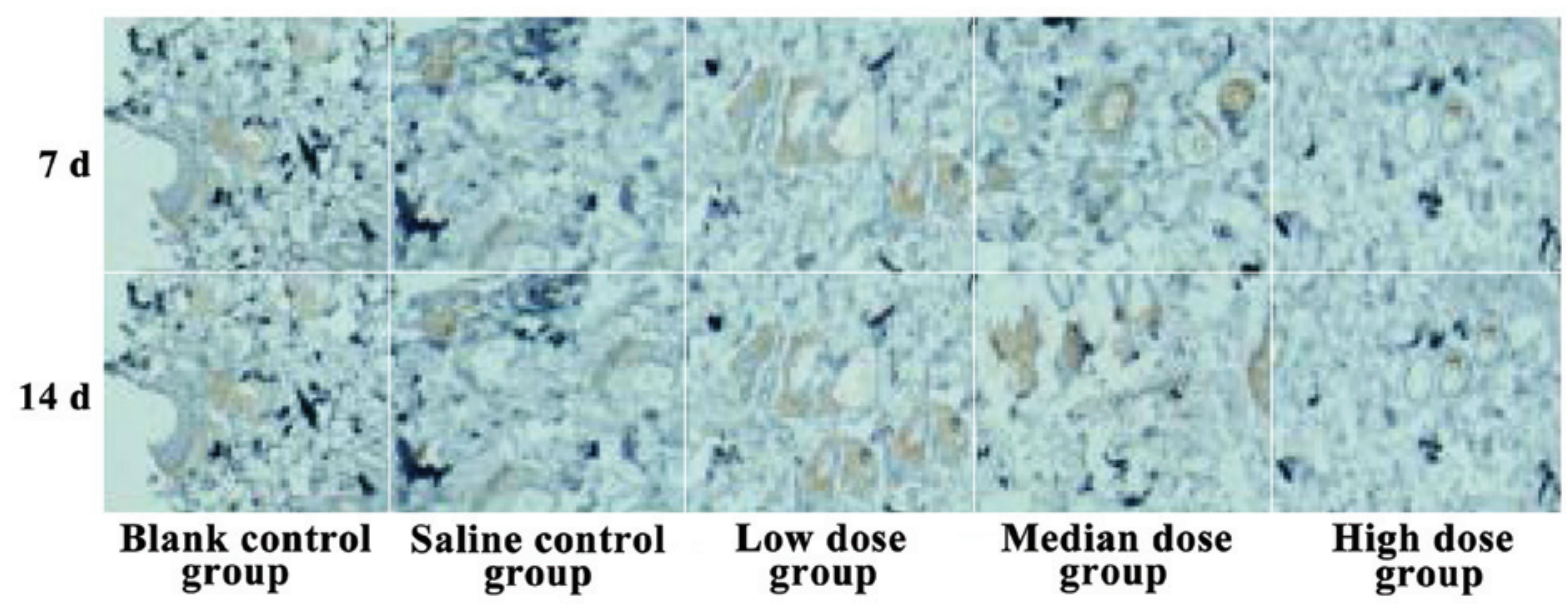

Figure 3. Microvessel quantity by immunohistochemistry using CD31 antibodies. Microscopic (magnification, x40) view of representative biopsy tissues from each group at 7 and 14 days.

differences in the blank control group, saline control group and high-dose group were not obvious (Table III).

Comparison of microvessel quantity. The microvessel quantities of the low-dose insulin group were the highest of them all at 14 days, while the quantities in the high-dose insulin group were the lowest. The differences found were statistically significant ( $\mathrm{P}<0.05$, Fig. 3).

Comparison of blood sugar level. The plasma glucose levels in patients gradually declined, and comparative differences over time were statistically significant $(\mathrm{P}<0.05$; Table IV).

\section{Discussion}

It is possible that the local insulin injections promote wound healing by stimulating the expression of VEGF, which in turn increases the number of blood capillaries. Additionally, glucose and amino acid entry into cells and the acceleration of protein synthesis, with increased fat synthesis and steatolysis inhibition promote wound granulation (6). Furthermore, the increase in the expression of epidermal growth factor and basic fibroblast growth factor in
Table IV. Comparison of blood sugar level ( $\mathrm{mmol} / \mathrm{l})$.

\begin{tabular}{lccc}
\hline Group & Before treatment & 7 days & 14 days \\
\hline Blank control & $7.6 \pm 1.5$ & $7.1 \pm 1.3$ & $6.3 \pm 1.1$ \\
Saline control & $7.7 \pm 1.4$ & $6.9 \pm 1.6$ & $6.4 \pm 1.3$ \\
Low dose & $7.5 \pm 1.5$ & $7.0 \pm 1.4$ & $6.5 \pm 1.2$ \\
Medium dose & $7.5 \pm 1.3$ & $6.8 \pm 1.2$ & $6.3 \pm 0.9$ \\
High dose & $7.6 \pm 1.4$ & $6.5 \pm 1.1$ & $6.1 \pm 1.6$ \\
F & 0.625 & 4.625 & 4.967 \\
P-value & 0.754 & 0.063 & 0.052 \\
\hline
\end{tabular}

wound tissues promotes fibroblast proliferation, increasing the hydroxyproline content in wound tissue, advancing the epidermal cell proliferation cycle and increasing synthesis of collagen fibers in the wounded tissue (7). The initial stage of repair and the proliferation of apoptosis after deep burns has the following adverse effects: Growth factor expression is weakened, granulation of the wounded tissues is not normal and consequently the survival rate of free flaps is not 
high due to the lack of a nurturing environment. Previous experiments conducted on animals have revealed that local application of low-dose insulin can promote wound healing in a diabetic rat model with scalds (8). High glucose conditions may lead to suppression of vascular endothelial cell proliferation, weakened cell contacts and increased apoptosis of the cells. At the same time, the decreased expression of VEGF delays the formation of new vessels, and the accumulation of advanced glycosylation end products leads to an extended inflammatory response and restrained collagen synthesis. The resulting microcirculation disturbance results in problems, such as insufficiency of local blood supply and local neurotrophic disturbances.

Whether the insulin is injected via subcutaneous, infiltration or intravenous injection, its actions depend on its ability to regulate the blood sugar metabolism. Insulin is a polypeptide with a short half-life in vivo. It may be decomposed by proteases in tissue when directly applied to wounds, and therefore reapplications are needed for treatment. The problem of how to continually release insulin in an effective manner to promote the growth of granulation tissue and wound healing is a known concern (9). Previous findings have shown that local infiltration injections increase the absorption efficiency of insulin while exerting little influence on blood sugar contents (10). The possibility that insulin may promote wound healing following simple deep burn skin flap transplantation in humans and its possible mechanism were analyzed in this clinical case-control study. Regular insulin was chosen because of its higher purity, easy absorbability, fast actions, and infimal probability of causing anaphylactic reactions (11). From the research findings it may be concluded that the wound healing rate of the low-dose insulin group significantly improved, the wound healing time shortened, the flap survival rate increased and the wound blood flow volume increased. Re-epithelization in the low-dose insulin group was faster, the infiltration of inflammatory cells was reduced, and fibroblast and new blood vessel counts were increased. The high-dose insulin group had the lowest wound healing rate, longest wound healing time and lowest total survival rate of flap of all of the groups in the study. By contrast, the mediumdose insulin group values were also better than the control group values. This suggests that the insulin effect on wound healing is related to its concentration. When the concentration is increased above a certain threshold, the effect is weakened, probably restraining normal wound healing speed. From this, it seems clear that a low-dose insulin can promote the regeneration of wound tissue $(12,13)$.

Expression levels of HSP-90, VEGF, TGF- $\beta$ and IL-1 were highest after 7 days in the low-dose insulin group, and the medium-dose insulin group took the second place. The comparative differences of the other 3 groups were not significant.

After all the biological cells including prokaryotic and eukaryotic cells were stimulated, the HSP-90 is a protein released in response to factors such as increased temperature, pathogen invasion, ischemia, hypoxia and contact to physical-chemical elements in different tissues, it is thought to protect cells from harmful stimuli. HSP-90 can promote wound healing, decrease ischemia reperfusion injury in flaps and increase survival rate of transplanted flaps (14).
VEGF is an important regulatory factor for new vessels and the strongest mitogen, in the growth factor family, that promotes endothelial cell proliferation. It also increases the permeability of capillaries, thus promoting the formation of new blood vessels (15). TGF- $\beta$ mainly comes from blood platelets, it can promote the chemotaxis, migration, proliferation, and differentiation of cells and the formation of granulation tissue by extracellular accretion and secretion, essential factors of wound healing $(16,17)$. IL-1 makes the generation of cells, neutrophils and lymphocytes possible by chemotaxis. It stimulates the fibroblasts to produce collagen and controls the formation of scarring tissue, playing a crucial role in wound healing. Local low-dose insulin acts independently of blood sugar metabolism in promoting wound healing, and it plays an important regulatory role in the course of regeneration of cell factors, growth factors and inflammatory reactions of multiple epithelia. Accordingly, this study showed that while the microvessel quantity of low-dose insulin group increased, the blood sugar levels did only slightly fluctuate.

In conclusion, the local application of low-dose insulin can markedly promote wound healing and the survival of transplanted flaps after operation for deep burns, and this is probably associated with the stimulation of higher expression levels of HSP-90, VEGF, TGF- $\beta$ and IL-1, which nevertheless, exert little influence on systemic blood glucose. Our approach has clinical practical value, and should be tested using similar clinical control studies with a larger population of patients.

\section{Acknowledgements}

The present study was supported by the Natural Science Foundation of China (no. 81071552).

\section{References}

1. Yu G, Ye L, Tan W, Zhu X, Li Y and Jiang D: A novel dermal matrix generated from burned skin as a promising substitute for deep degree burns therapy. Mol Med Rep 4: 23-25, 2016.

2. Lei J, Hou C, Duan P, Hao Z, Zhai Y and Meng Y: Clinical application of modified skin soft tissue expansion in early repair of devastating wound on the head due to electrical burn. Zhonghua Shao Shang Za Zhi 31: 406-409, 2015 (In Chinese).

3. Chi Y, Chai J, Xu C, Luo H and Zhang Q: Apelin inhibits the activation of the nucleotide-binding domain and the leucine-rich, repeat-containing family, pyrin-containing 3 (NLRP3) inflammasome and ameliorates insulin resistance in severely burned rats. Surgery 157: 1142-1152, 2015.

4. Wang M, Xue X, Xie P and Zhang J: Effects of nerve growth factor-insulin composite gel on deep second degree scald wound healing in diabetic rats. Zhongguo Xiu Fu Chong Jian Wai Ke Za Zhi 27: 182-188, 2013 (In Chinese).

5. Bircher A, de Boer EM, Agner T, Wahlberg JE and Serup J: Guidelines for measurement of cutaneous blood flow by laser Doppler flowmetry. A report from the Standardization Group of the European Society of Contact Dermatitis. Contact Dermatitis 30: 65-72, 1994.

6. Dong J, Tang Q, Li W and Tian F: Our preliminary experiences in topical administration of insulin in addition to vacuum assisted closure for wound healing in diabetic patients. Minerva Chir 70: 389-391, 2015.

7. Kim SM, Kim YH, Jun YJ, Yoo G and Rhie JW: The effect of diabetes on the wound healing potential of adipose-tissue derived stem cells. Int Wound J 13 (Suppl 1): 33-41, 2016.

8. Salazar JJ,Ennis WJ and Koh TJ: Diabetes medications: Impact on inflammation and wound healing. J Diabetes Complications 30: 746-752, 2016 
9. Dhall S, Silva JP, Liu Y, Hrynyk M, Garcia M, Chan A, Lyubovitsky J, Neufeld RJ and Martins-Green M: Release of insulin from PLGA-alginate dressing stimulates regenerative healing of burn wounds in rats. Clin Sci (Lond) 129: 1115-1129, 2015.

10. Azevedo F, Pessoa A, Moreira G, Santos MD, Liberti E, Araujo E, Carvalho C, Saad M and Lima MH: Effect of topical insulin on second-degree burns in diabetic rats. Biol Res Nurs 18: 181-192, 2016.

11. Attia EA, Belal DM, El Samahy MH and El Hamamsy MH: A pilot trial using topical regular crystalline insulin vs. aqueous zinc solution for uncomplicated cutaneous wound healing: Impact on quality of life. Wound Repair Regen 22: 52-57, 2014

12. Zhang XJ, Meng C, Chinkes DL and Herndon DN: Beneficial effects of insulin on cell proliferation and protein metabolism in skin donor site wound. J Surg Res 168: e155-e161, 2011.

13. Zhang XJ, Wu X, Wolf SE, Hawkins HK, Chinkes DL and Wolfe RR: Local insulin-zinc injection accelerates skin donor site wound healing. J Surg Res 142: 90-96, 2007.
14. Woodley DT, Wysong A, DeClerck B, Chen M and Li W: Keratinocyte migration and a hypothetical new role for extracellular heat shock protein 90 alpha in orchestrating skin wound healing. Adv Wound Care (New Rochelle) 4: 203-212, 2015.

15. Puddu A, Sanguineti R, Traverso CE, Viviani GL and Nicolò M: Response to anti-VEGF-A treatment of endothelial cells in vitro. Exp Eye Res 146: 128-136, 2016.

16. Kryger ZB, Sisco M, Roy NK, Lu L, Rosenberg D and Mustoe TA: Temporal expression of the transforming growth factor-Beta pathway in the rabbit ear model of wound healing and scarring. J Am Coll Surg 205: 78-88, 2007.

17. Koskela von Sydow A, Janbaz C, Kardeby C, Repsilber D and Ivarsson M: IL- $1 \alpha$ counteract TGF- $\beta$ regulated genes and pathways in human fibroblasts. J Cell Biochem 117: 1622-1632, 2016. 